\title{
Watchdog: A Novel, Accurate and Reliable Method for Addressing Wandering-off using Passive RFID Tags
}

\author{
Rengamathi Sankarkumar, Damith C Ranasinghe \\ Auto ID Lab \\ The University of Adelaide \\ North Adelaide, Australia \\ rengamathi.sankarkumar,damith.ranasinghe@adelaide.edu.au
}

\begin{abstract}
Hospitals and residential homes have a significant need for monitoring and recognising wandering-off (e.g. elopement) older people with cognitive impairments because of the serious consequences arising from wandering-off such as disappearances and serious injuries, for example, from collisions with vehicles in parking lots. Due to increasing ageing populations across the globe we can expect wandering-off to become a significant problem of scale affecting all of us. Existing technologies used to address wandering-off are inadequate for providing close supervision as they use proximity based sensing that often lead to false alarms. In this study, for the first time, we try to mitigate false alarms by identifying the traversal direction and traversal path used by people instrumented with a single low cost batteryless UHF RFID tag. Our approach uses a particle filtering (PF) based technique with Received Signal Strength Indicator (RSSI) maps obtained from scene analysis to continuously track a person wearing an RFID tag over their attire. Using real-time spatial and temporal data obtained from the PF based tracking algorithm, we develop two algorithms: i) tag traversing direction (TD) algorithm to identify the tag bearer's moving direction (e.g. moving out of a room); and ii) tag traversing path detection algorithm (TPD) to estimate the traversal path used by the tag bearer. Our extensive experiments with 14 young adult volunteers show that: i) our TD algorithm can identify the moving direction of a person with $100 \%$ accuracy; ii) our TPD algorithm reduces the false alarms to $<$ $9 \%$, when detecting the traversing path used while eloping; and iii) our algorithms can be implemented in a different environment without further scene analysis.
\end{abstract}

\section{Categories and Subject Descriptors}

I.4 [Image Processing and Computer Vision]: Scene Analysis-Tracking; C.3 [Special-Purpose and Application - Based Systems]: Real-time and embedded systems

\section{Keywords}

Permission to make digital or hard copies of all or part of this work for personal or classroom use is granted without fee provided that copies are not made or distributed for profit or commercial advantage and that copies bear this notice and the full citation on the first page. To copy otherwise, to republish, to post on servers or to redistribute to lists, requires prior specific permission and/or a fee. MOBIQUITOUS 2014, December 02-05, London, Great Britain Copyright (c) 2014 ICST 978-1-63190-039-6 DOI 10.4108/icst.mobiquitous.2014.258040 wandering-off, RFID, tag traversing direction, traversing path detection, particle filter

\section{INTRODUCTION}

Wandering-off (e.g. elopement) [6] among older people with dementia, Alzheimer's disease (AD) and other cognitive impairments are common $[2,12,18,4]$. Wandering-off may result in serious consequences such as getting lost, collision with vehicles or even death and the responsible aged care service providers are liable for such actions [2]. Therefore, continuous monitoring among wandering-off patients is essential. Due to ageing populations around the globe, occurrences of wandering-off incidences are expected to increase. It is estimated that, by 2050 the number of people with AD is expected to be around 900,000 in Australia [2] and about 16 million in U.S. [2]. Monitoring and recognising these patients when they are exiting cared areas with a reliable system can minimise the occurrence and associated risks of wandering.

Current technologies [6, 25, 27], that address wanderingoff are mostly boundary alarms that use simple proximity based sensing. As a result, false alarm rates (i.e. incorrectly detecting that the person has walked through the doorway when they are still in the cared area) are high in these systems [6]; also, information about the traversal path used by the older person is non-existent. Using such technologies to provide continuous care to prevent wandering-off can be challenging, stressful and frustrating for caregivers [6].

RFID (Radio Frequency Identification) is an enabling technology in tracking applications. RFID is capable of automatically and uniquely identify individuals [9]. In this study, for the first time to the best of our knowledge, we introduce Watchdog, a system to mitigate false alarms by accurately and reliably identifying the traversal direction and traversal path used by people instrumented with a single passive RFID tag. Watchdog recognises the tag traversal direction and traversal path by utilising a Particle Filter (PF) with Received Signal Strength Indicator (RSSI) maps rssi_map obtained from the interrogation between an RFID reader and a passive tag. Detecting tag traversal direction and traversal path is vital among wandering-off older people because, the undesirable traversal direction of a person can be known by a caregiver, for example, if a caregiver is aware that a patient is moving out of their room, then the caregiver can stop them from elopement or go in search of them immediately after elopement. Furthermore, knowing the traversal 


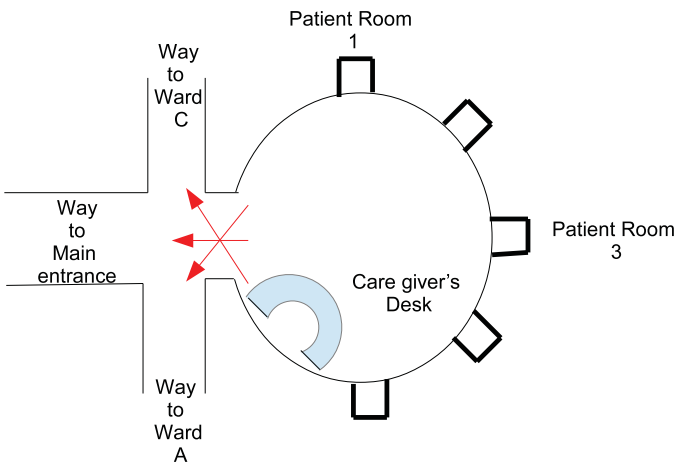

Figure 1: Example for probable direction of elopement of patients in a hospital setting

path can be helpful to reduce the search space and to estimate the level of risk associated while eloping. For example, as shown in Fig. 1, knowing the traversing path used by a patient is towards the right corridor, reduces the search space in the event of an elopement. Knowing the traversing path allows estimating the level of risk associated with an elopement and help prioritise the searching process. For instance in Fig. 1, if the patient has used a path forwards to the main entrance then the associated risk with this path is higher when compared to other paths. We summarise the main contributions of our paper as follows:

- We propose an accurate and reliable system, Watchdog, to determine wandering-off among older people wearing a low cost passive RFID tag attached over their attire to alert the caregivers with identified traversal direction and traversal path when the tag bearer approaches or exits a doorway.

- We develop two algorithms, namely, traversing direction (TD) detection algorithm and traversing path detection (TPD) algorithm to accurately identify the tag traversing direction and tag traversing path, respectively, used by the tag bearer from the spatial and temporal data obtained from a real-time PF based technique.

- Finally, we implement the algorithms in two environments (a supervised environment and an unsupervised but similar environment) and conduct extensive experiments with 10 volunteers to evaluate the performance and accuracy of our proposed algorithms. We also compare the performance of our approach with traversing direction (TD) detection and traversing path detection with scene analysis and demonstrate the superior performance of Watchdog.

\section{RELATED WORK}

For a clearer overview we divide our discussion into three parts. Firstly, we discuss some of the existing methods employed to prevent wandering-off. Secondly, we discuss literature that identify tag traversing direction, thirdly, we discuss the traversal path detection techniques used with networked RFID and finally, we discuss existing localisation methods.

\subsection{Overview of Existing Alarm Methods}

Uses of alarms on door exits is one of the well known technologies in monitoring older people. There are two types of alarm systems [6]: i) alarms that sounds when the door is opened; and ii) alarms that sound when a person wearing a sensor (e.g. a battery powered wrist bracelet) approaches the door. However, these kinds of alarms have several drawbacks such as caregivers not hearing the alarm, inability to immediately identify the location of the alarm, older people removing the bracelet or battery of the worn device being flat $[6,21]$. Some of the recent researchers have used android powered phones [25] and battery powered WiFi tags [27] to address wandering. However, a common drawback for all the above mentioned technologies is the need to carry bulky battery powered devices. Furthermore, in systems that sound an alarm when doors are opened, automatically and uniquely identifying an individual is still a challenging task because door alarms sound when a person enters its readable range and are not capable of differentiating caregivers from patients. Therefore, even in case of hearing an alarm, there is negligence among caregivers as alarms are assumed to be triggered by a carer [6]. In contrast to existing methods our PF based algorithms for continuous monitoring of older people are robust and accurate and thus capable of drastically reducing false alarms.

\subsection{Traversing Direction and Traversal Path Detection}

Number of works that utilise passive tags for determining tag traversal direction are limited. In [13], authors use several antennas and record the tag events as they are detected. Then using the order of events, tag direction is determined. However, their research is conducted using relatively more expensive active (battery powered) RFID tags to determine the traversing direction of a tag. In [20], time intervals between tag detections by static reader antennas are used to find the tag traversal direction, however, this method has only been successful with dense tags (10 or more) and cannot be implemented with single tag. In [30], direction of arrival (DoA) is used to find the moving direction of a tag, however, real-time evaluation of this method is not reported in the paper. In [32], we developed two methods using tag phase and its radial velocity to determine the direction of a passive tag worn by a person. However, the accuracy of identifying the tag traversal direction is less than $90 \%$ and it is also likely to be adversely affected by higher walking speeds of a tag bearer.

To the best of our knowledge, we are the first to study traversing path of a tag bearer using passive RFID tags attached to their outfit using fixed antennas. Although mobile robots' trajectories were investigated in $[14,11]$ by utilising mobile antennas and fixed tags, mobile robots are mounted with RFID antennas and their trajectories are determined from the location of static (fixed) tags attached to walls. These techniques relies on dense tag deployments on walls to determine the trajectory used by the robot and have been specifically designed for scenarios such as stock taking in supermarkets [14] where static tags are placed on shelving. If these approaches are directly implemented in our problem context then more resources are needed than what we currently use, for example, multiple tags have to be attached to 


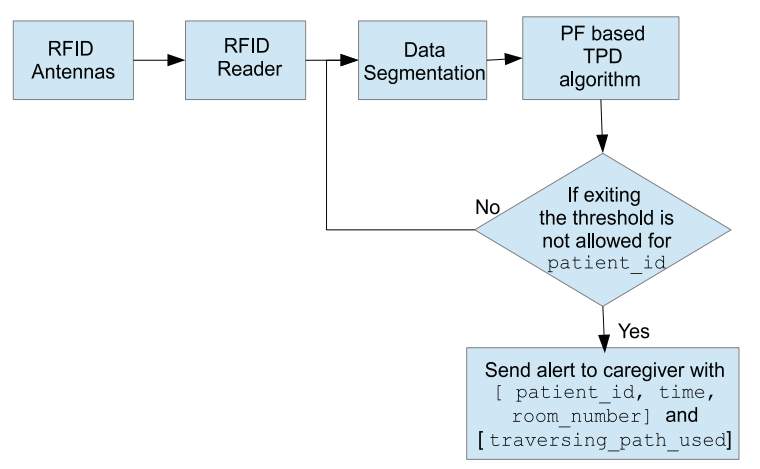

(a)

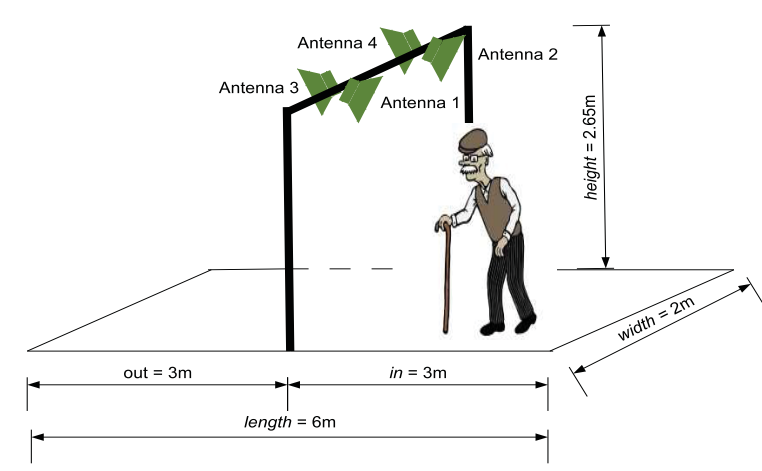

(b)

Figure 2: (a) System overview (b) An example setup

the ground over the monitoring area. Also, patients have to carry wrist worn battery powered RFID readers [21] instead of low cost, lightweight and batteryless tags. In contrast, our developed algorithms are capable of accurately and reliably identify the traversal direction and path used by a person instrumented with a single passive RFID tag.

\subsection{Localisation Methods}

Nevertheless, a number of localisation methods exists that can be used to infer the tag bearer's location. These RFID based localisation techniques can be broadly classified into three main categories [8]:

1. Distance based estimation: This kind of estimation depends upon the use of properties of triangles such as triangulation and trilateration $[8,16]$. The range measurement parameters are obtained from Received Signal Strength Indicator (RSSI) [10], Time of Arrival (ToA) [13], Angle of Arrival (AoA) [28], Time Difference of Arrival (TDoA) [20] and Received Signal Phase (RSP) [32].

2. Proximity based estimation: Proximity based estimation is a kind of sensing technique which determines how close an object is from a known priori location. If a tag is detected by a reader antenna then the location of the tag is assumed to be within the readable zone of that particular antenna [16].

3. Scene analysis: Scene analysis consists of two distinct steps $[10,19,31,22]$. In step 1 , information about the features of the environment is collected and in step 2 , obtained real-time measurements are compared with the previously collected data (from step 1) to infer the current location of the object.

In [28], authors introduce a robust, fine grained RFID tag positioning system that utilises AoA and proximity based localisation. In addition to these techniques, $k$-NN algorithm is used to infer the desired tag location from the nearest reference tag. Passive RFID tags are used for both the desired and reference tags. Landmarc [19] utilises scene analysis technique to identify the spatial position of a desired tag from reference tags with known locations. They first locate the reference tags that are near to the desired tag and then using the RSSI values and $k$-NN algorithm, the nearest tag location is calculated. Some of the other works that utilise scene analysis to localise the desired tag location with the help of reference tags are discussed in [31, 22, ?]. In [31], the authors localise the desired tag's location by utilising a 2D grid of reference tags and a proximity map, while in [22], kalman filter based technique is used in locating the desired tag and in [?] weighted centroid localisation and PF are employed to track the objects. However, all the above discussed methods, regardless of the technique they use, rely on reference tags to localise the position of the desired tag.

In [29] indoor spatial queries are evaluated from a PF based method. In contrast to other studies discussed, this work does not need reference tags but introduces nodes and edges all along the state space and assume that the object is moving only along the nearest edge by compromising on finegrain localisation. Also, the discontinuity in their antenna setup does not allow continuous tracking of objects, instead, objects missing over a period is assumed to be in one of the rooms that is nearest to the last seen location. Although such methods can be beneficial in a estimating spatial queries, it cannot be directly implemented in determining the traversal direction and path. However, the research methods used in [29] serves as a basis for our work which also does not rely on reference tags. In contrast to [29] we are interesting in continuous and accurate monitoring of temporal and spatial coordinates of a tag bearer. In particular, our Watchdog system is capable of identifying the tag traversing direction (e.g. moving out of a room) and tag traversal path from the raw RSSI readings obtained from a passive tag by tracking the tag in real time and preserving the information gathered in the past.

Except [29], all other localisation techniques discussed above successfully localise a tag using more expensive active RFID tags, in contrast, we use low cost, lightweight, passive (batteryless) RFID tags which power themselves when they are interrogated by an RFID antenna. Therefore the received signals in our system are often noisier and can only be used in a limited working range. We are interested in using passive RFID tags because they are maintenance free (batteryless), unobtrusive and can be easily integrated into clothing as washable passive RFID tags are already a commercial re- 


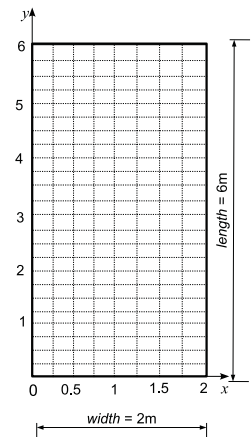

(a)

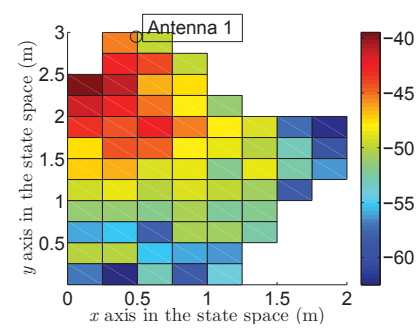

(b)

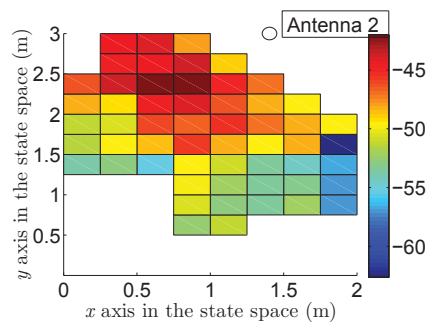

(c)

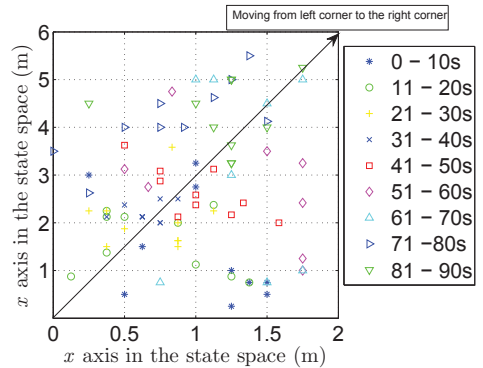

(d)

Figure 3: (a) State Space for scene analysis (b) rssi_map for Antenna 1 (c) rssi_map for Antenna 2 (d) Scene Analysis

ality [3]. Also, hospitals are places where hygiene is a top priority, so these low cost tags can be easily disposed. In the next section we discuss an overview of our system.

\section{AN OVERVIEW OF WATCHDOG}

We introduce Watchdog, a real-time algorithm capable of reliably identifying the traversal direction and traversal path used by a person wearing a passive RFID tag on their attire. We named our approach Watchdog, because usually watch dogs are trained to protect people from hazards. Also their keen sense of smell is capable of identifying the traversing path used by a particular person. Fig. 2(a) depicts an overview of our system and Fig. 2(b) shows an experimental setup of our system. The components we used in Watchdog to address wandering off are: i) a four port RFID reader; ii) four RFID antennas; iii) a passive RFID tag attached over clothing and iv) two algorithms: detecting the tag traversal direction and the traversing path used while eloping.

When a passive RFID tag enters the monitoring zone, the 4 mounted RFID antennas power and interrogate the tag in order to obtain time of the read, EPC (Electronic Product Code) assigned for each person, antenna ID that identified the the antenna reading the tag and the RSSI value. Thus, raw RFID reads $r$ obtained here can be represented by the schema: $\left[t\right.$, patient $_{I D}$, ant, rssi]. In contrast to the existing RFID localisation systems, Watchdog accurately identify the traversal path used by a tag bearer without any reference tags deployed in the state space. Instead, Watchdog employs an rssi_map that depicts the state space features through a scene analysis technique [10]. Further, Watchdog enhances the system by utilising the spatial and temporal data obtained from the PF because scene analysis is prone to noise in RSSI measurements that can lead to location uncertainty.

The following sections of the paper are summarised as follows: Section 4, describes scene analysis to acquire rssi_map individually, for each antenna deployed in the state space and provides an overview of a baseline method using scene analysis to determine TD and TPD; Section 5 describes the PF based algorithms employed by Watchdog to to determine TD and TPD; Section 6 presents the experimental evidence to demonstrating the performance of our Watchdog system; and we conclude our article in Section 7 .

\section{SCENE ANAYSIS}

Before explaining the techniques involved in Watchdog we perform scene analysis in the monitoring zone. First, we partition the 2-D state space equally where the dimensions of a partition is approximately equal to a walking step. We denote the intersection of each partition with their $x$ and $y$ axis as $(x, y)$.

As shown in Fig. 3(a) the state space used in our experiment is $6 \mathrm{~m}$ in length and $2 \mathrm{~m}$ in width, where we considered the width of the state space along the $x$ axis and the length along the $y$ axis. We divided the space in such a way that each partition measures $25 \mathrm{~cm}$ in length and $25 \mathrm{~cm}$ in width. We ask a tag bearer to stand static in each of these intersections for approximately 4 seconds. Then, we calculate the mean of the obtained RSSI values and generate an rssi_map for each individual antenna deployed in the state space. For example, Fig. 3(b) and (c) show the rssi map for the two antennas deployed in the inner side in our experiment scenario shown in Fig. 2(b). The combined collected rssi_map obtained from all the antennas deployed reveals the RSSI features over the state space.

\subsection{TD and TPD with Scene Analysis}

We employed scene analysis based approaches described in [10, $19,31,22]$ to serve as a baseline for evaluating Watchdog. We utilise the scene analysis technique to detect the tag traversal direction and tag traversal path. We partition a sequence of tag reading $r_{1: m}=\left\{\left(t_{i}, r s s i_{i}, a n t_{i}\right)\right\}_{i=1}^{m}$ in a non-overlapping fixed time segment $\delta t$ for a given patient $t_{I D}$ where $t$ is the time stamp of a tag read, rssi is the Recertify Signal Strength Indicator value and ant is the ID of the antenna that captured the tag read at time $t$. From the sequence $r_{1: m}$, we obtain the observation $z_{t}$, by calculating the mean RSSI value $\overline{r s s i}_{a n t}$ for each antenna ant that obtained a tag read.

$$
z_{t}=\left\{\overline{r s s i}_{\text {ant }}\right\}_{\text {ant }=1}^{a n t=w}
$$

where, the first time stamp $t_{1}$ in $r_{1: m}$ is used as the time $t$ for the observation $z$ and $w$ denotes the number of antennas that captured a tag response in the sequence $r_{1: m}$. The 


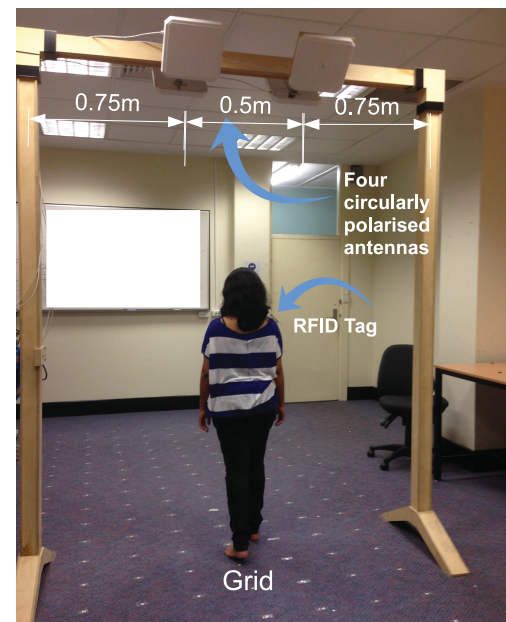

(a)

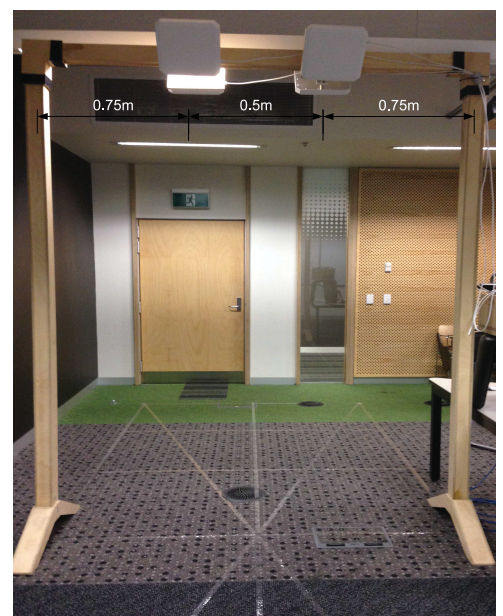

(b)

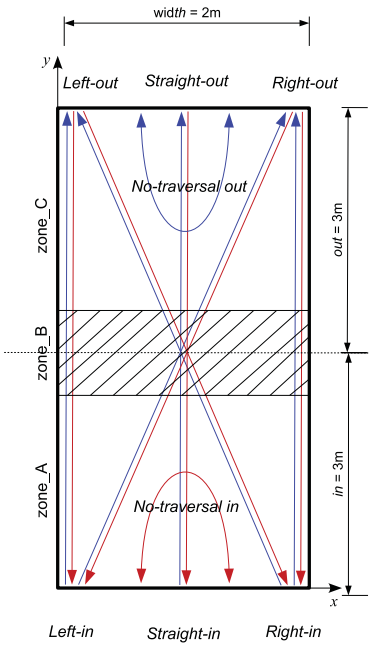

(c)

Figure 4: (a) Supervised environment (b) Unsupervised Environment (c) Traversal path evaluated in the experiments

obtained mean RSSI value $\overline{r s s i}_{a n t}$ is compared with the previously developed rssi_map to infer the tag bearer's location and then subsequently determine TD and TPD.

Fig. 3(d) shows the result of directly comparing the $\overline{r s s i}_{\text {ant }}$ with the acquired rssi_map to identify the tag traversal path from the left corner of the state space to right corner. From Fig. 3(d) it is clear that readings from the passive RFID tags are highly noisy and no exact inference about the tag traversal path can be made with just scene analysis. Therefore in the next section we introduce a PF based TD and TPD algorithms to overcome this location uncertainty.

\section{PF BASED TD AND TPD ALGORITHM}

A particle filter (also known as sequential monte carlo) works in a recursive fashion to estimate the posterior distribution of a hidden state (e.g., location of a patient) using the observations obtained (e.g., RSSI value) from the measurement process [5]. Our approach is capable of continuously tracking the tag location with information about the tag traversing direction. Our approach is also capable of overcoming missed reads (false negatives) in the real time RFID data, which are usually quite common in raw RFID data $[23,24]$. Next, we explain the two critical models that are used to infer the location of an object (e.g. tag) in a dynamic system. They are: i) motion model; and ii) sensor model.

Motion model: Motion model or system dynamics describes how the system evolves from the time step $t-1$ to the time step $t$.

$$
l_{t}=f_{t}\left(l_{t-1}, v_{t-1}\right)
$$

where $l_{t}$ is the true state of the tag, and $v_{t-1}$ is independently and identically distributed (i.i.d.) process noise.

$$
p\left(l_{t} \mid l_{t-1}\right)=p\left(l_{t} \mid s, \theta, l_{t-1}\right)
$$

The motion model used in our system is shown in (2), where $l=(x, y)$ is the coordinate revealing the state of a tag. The conditional probability $\mathrm{p}\left(l_{t} \mid s, \theta, l_{t-1}\right)$, specifies the possible motion of the tag from the previous iteration to the current iteration, given the learning velocity factors: speed, $s$; and direction, $\theta$. We have considered building a model that can dynamically adapt to the walking speed and direction of a person. Initially we considered the moving speed $s$ to be the mean gait speed reported in [7] for people aged 40 and above and the probability of moving in any of the given direction $\theta$ to be equiprobable where $\theta=\left\{0^{\circ}, 45^{\circ}, 90^{\circ}, \ldots\right.$, $\left.315^{\circ}\right\}$. After every iteration we consider the difference between the predicted location and the estimated location to additively increase the speed $s$ to adapt to increasing walking speeds and multiplicatively decreasing speed to adapt to the decreasing walking speeds and halts. The direction $\theta$ is updated by multiplicative increases of the probability in the direction of traversal in the previous time $t-1$ and decreases in the probability of moving in all other directions.

Sensor model: Once there is an observation the measurement model describes how the observation $z_{t}$ relates to the true state $l_{t}$ of the system.

$$
z_{t}=h_{t}\left(l_{t}, u_{t}\right)
$$

where $h_{t}$ is a possible non-linear function, and $u_{t}$ is i.i.d. measurement noise.

$$
p\left(z_{t} \mid l_{t}\right)=p\left(\overline{r s s i}_{a n t} \mid l_{t}, \text { ant }, \text { rssi_map }\right)
$$

The sensor model $p\left(z_{t} \mid l_{t}\right)$ used in our system specifies the likelihood of obtaining a measurement $z$ given the true state of the tag $l_{t}$. The probability of having a mean RSSI $\overline{r s s i}_{\text {ant }}$ for a given predicted location $l_{t}$, antenna ID ant and the corresponding map rssi_map for ant is determined in our sensor model. Below we discuss briefly the steps involved in one iteration of the PF.

Initialize: The given particles are first initialised for the state $l$. At time step $t=0$, for $N=1, . ., n$ sample the state particles, $l_{0}^{N} \sim p\left(l_{0}\right)$

Predict: Using the motion model predict the location of 


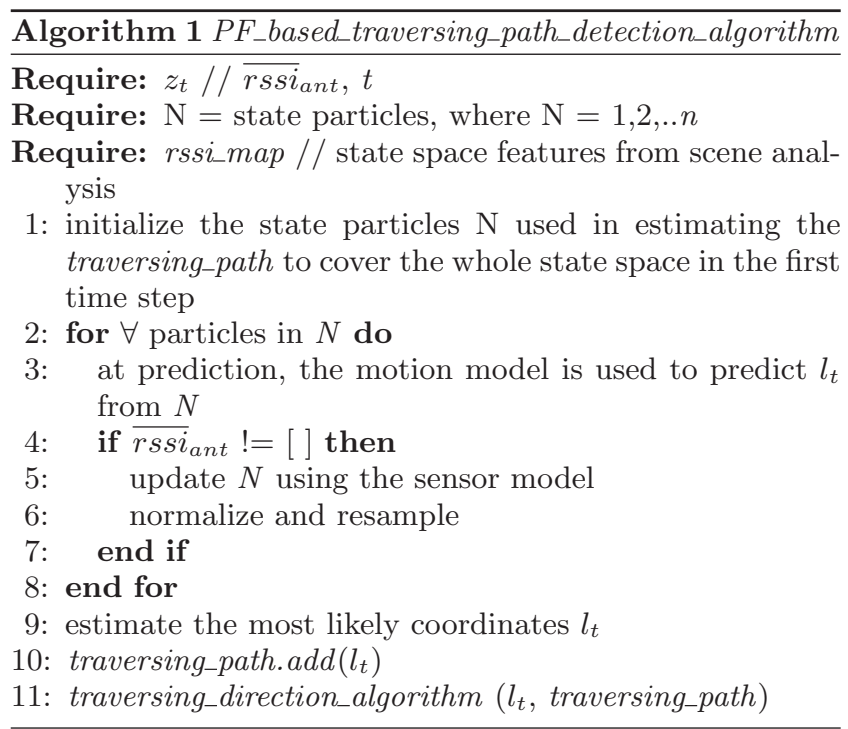

the object at each time step. At $t$ the state l's particles are predicted to be in a location considering the state at $t-1$ and the observations $(z)$ obtained so far. For $N=1, \ldots, n$, predict particles, $l_{t}^{N} \sim q\left(l_{t} \mid l_{t-1}^{N}, z_{1: t}\right)$, where $\mathrm{q}($.$) is an importance$ function [5].

Update: On receiving an observation $z_{t}$, the predicted particles' locations are updated by weighting the particles using the measurement model to obtain importance weights $w_{t}$, $w_{t}^{N}=p\left(z_{t} \mid l_{t}^{N}\right)$, where high weights are given to the particles nearer to the measurement.

Normalize: The particle's weight are normalised. For $N=$ $1, \ldots, n$, normalize the importance weight, $w_{t}^{N}=w_{t}^{N} / \sum_{j=1}^{n} w_{t}^{j}$.

Resample: Increasing number of PF iterations leads to sample degeneracy, which means only few particles would have non-negligible weights while the remaining would have near-zero weights [5]. The resampling step eliminates the lower weighted particles and replicates higher weight particles to generate a new set of particles with equal weights [29]. The new set of particles thus obtained is equal to the original number of particles. For $N=1, \ldots, n$, set, $w_{t}^{N}=1 / n$.

\subsection{Traversing Path Detection Algorithm}

We detect the traversing path used by the tag bearer using a PF based TPD algorithm with prior knowledge of the state space features. Algorithm 1 is called with the observation $z$ with mean RSSI $\overline{r s s i}_{a n t}$ at $t$. In line 1 , we initialise the $N$ particles used in estimating the traversing path. In line 3 , we predict the possible current locations from the motion model. Line 4, checks whether the $\overline{r s s i}_{\text {ant }}$ is empty, in order to identify missed reads.

If there is a missed read in the real-time data, i.e., no reading was obtained in time $\delta t$, then our TPD algorithm simply predicts the current location and conclude the estimation without updating and resampling. This is because update and resample steps are only necessary when there is an observation (see lines 5 to 8 ). In line 9 , we finally estimate the $(x, y)$ coordinates.

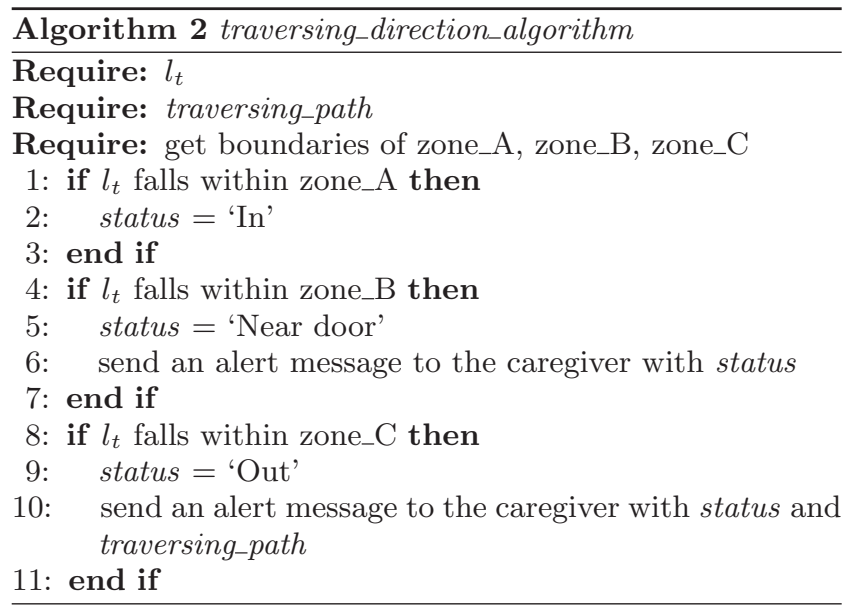

\subsection{Traversing Direction Algorithm}

In this section we explain the traversing direction algorithm. From the estimated $(x, y)$ coordinates from $l_{t}$ obtained from the PF based TPD algorithm we find the tag traversing direction. We classify the state space into three categories to implement the TD algorithm: i) zone_A is the space that is covered uniquely by the antennas that are deployed in the inside region of the state space; ii) zone_B is the space where the reading zone of antennas deployed inside and outside overlap; and iii) zone_C is the space that is covered uniquely by the antennas that are deployed in the outside region of the state space. Our Algorithm 2, classifies the estimated location $l_{t}$ of the tag bearer to a corresponding zone. If the tag bearer is found approaching or entered the disallowed zones an alert is given to a caregiver with the direction status and the traversal path used by the tag bearer traversing_path (see Algorithm 2).

\section{EXPERIMENTS AND RESULTS}

We conducted extensive experiments in two laboratory environments (supervised and unsupervised) to evaluate the ability of our algorithms to accurately identify the traversing path and the traversal direction used by the tag bearer. We further compare the results of our PF based TD and TPD algorithm with the scene analysis technique described in Section 4 as a baseline.

\subsection{Settings and Data Collection}

An overview of our system is shown in Fig. 3(a) and the experimental setup is shown in Fig. 4. The state space includes an area with length $=6 \mathrm{~m}$, width $=2 \mathrm{~m}$ and height $=2.65 \mathrm{~m}$ from the ground level. We considered the wooden frame (shown in Fig. 4) of $2 \mathrm{~m}$ width and $2.65 \mathrm{~m}$ height as the threshold that partitioned the inside (cared area) and the outside. Two antennas were deployed on inner side of the frame and two were deployed on the outer side. The antennas were located $0.75 \mathrm{~m}$ from the side of the frame. All four antennas were inclined at $45^{\circ}$ because a better illumination of the state space was obtained at this angle. The four antennas employed are circularly polarised antennas of model no: Impinj IPJA1000-USA. We used an Impinj Speedway Revolution UHF (Ultra High Frequency) RFID reader (R420) and 'Squiggle' passive tags. 


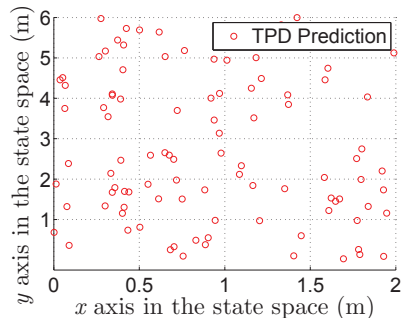

(a)

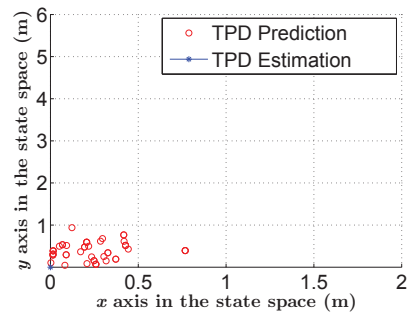

(b)

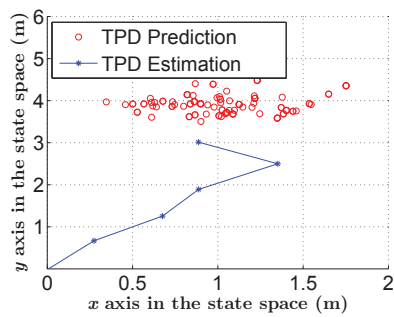

(c)

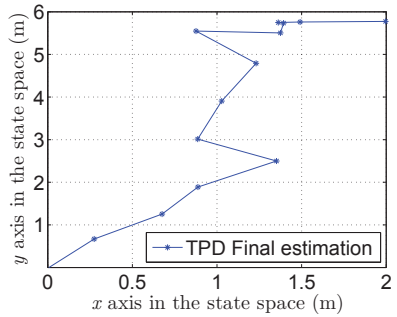

(d)

Figure 5: (a) Initialisation (b) At first observation (c) Eloped from the secured caregiving area but still in the reader detectable area (d) After complete elopement in the Right out direction

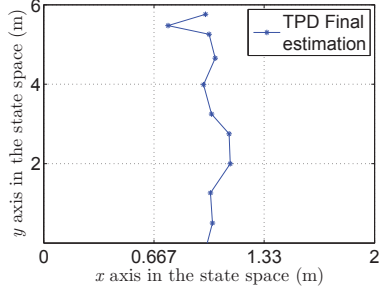

(a)

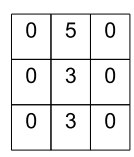

(i)

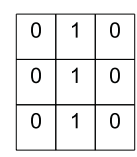

(ii)

(b)

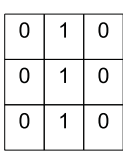

(iii)

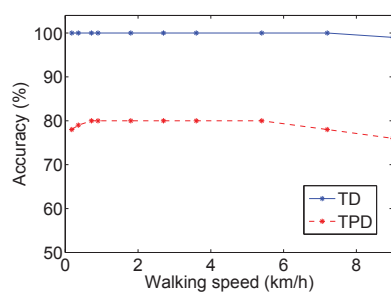

(c)

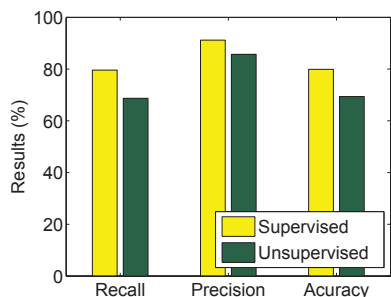

(d)

Figure 6: (a) Effects of number of particles used in PF (b) Accuracy in terms of speed (c) Comparison of our algorithm results with the supervised environment and a unsupervised environment

We considered 12 paths as shown in Fig. 3(c) where Rightout to Left-in, Right-out to Right-in, Straight-out to Straightin, Left-out to Left-in and Left-out to Right-in were considered as moving in paths and Left-in to Right-out, Left-in to Left-out, Straight-in to Straight-out, Right-in to Left-out and Right-in to Right-out were considered as moving out paths. Two non-traversal paths, namely No-traversal out and Notraversal in were included to consider situations that involve activities inside the room or simply walking towards the outside and turning back.

We conducted our experiments in two environments, namely, supervised (where scene analysis was conducted) and unsupervised (a new and different environment without where scene analysis was not performed) as shown in Fig. 4(a) and (b), in order to evaluate the robustness of our algorithms in a different environment and to assess the ability to use the noise model developed through scene analysis in the supervised environment in a completely new environment. Our participants performed a routine of 25 moving in path trials (a list of 25 paths randomly selected from moving in paths), a routine of 25 moving out path trials ( 25 randomly selected from moving out paths) and 20 non-traversing path trials.

Fourteen healthy, young adults aged between 25 to 35 participated in the laboratory experiments. The mean \pm SD height of our participants was $169 \pm 8 \mathrm{~cm}$. However, only the first 6 participants were involved in both the experiments the last 4 participants were different in the supervised and unsupervised environment experiments. The passive RFID tag was attached over each participant's attire using a double sided adhesive tape over the right shoulder as shown in Fig. 4(a). All the participants were asked to walk at their normal speed and were not instructed to walk at any specified speed.

\subsection{Illustration of the PF based TPD Algorithm}

Fig. 5(a-d) shows an step by step example of the TPD algorithm's prediction and final estimation for the path starting from Left-in to Right-out. At the initialisation step particles are distributed over the state space as shown in Fig. 5(a) since the location of the tag bearer is completely uncertain. Then, after an observation our algorithm predicted the tag bearer's location as shown in Fig. 5(b). Red circles in the figures denote the predictions of TPD in the current time step and blue asterisks denote the estimation of TPD. Fig. 5(c) shows the estimated traversing path of the tag bearer in further iterations. Fig. 5(d) shows the detected traversing path used after eloping. Considering our TPD algorithm's results in Fig. 5(d) with scene analysis results obtained in Fig. 3(d) which utilised the same path (Left-in to Right-out) and RSSI measurements to detect the traversing path clearly illustrates the robustness of approach even under highly uncertain RSSI measurements.

\subsection{Method for Evaluating the TPD Algorithm}

In order to evaluate the performance of our TPD algorithm we partition our state space $2 m \times 6 m$ into 9 equal partitions as shown in Fig. 6(a). We evaluated accuracy by counting the number of final estimation in each of these partitions. For example, in Fig. 6(a) we evaluate the Straight-in to Straight-out path. Here counting the number of location estimations in each partition is as indicated Fig. 6(b)(i). From this result we determine the maximum occurrence in each 
Table 1: Performance of our PF based algorithms in a supervised environment and comparison with the scene analysis technique

\begin{tabular}{|c|c|c|c|c|c|c|c|c|}
\hline Person & Path & TP & FN & TN & FP & Recall & Precision & Accuracy \\
\hline \multirow[t]{2}{*}{ Person 1} & Moving in & 19 & 6 & 8 & 2 & $76 \%$ & $91 \%$ & $77 \%$ \\
\hline & Moving out & 21 & 4 & 8 & 2 & $84 \%$ & $91 \%$ & $83 \%$ \\
\hline \multirow{2}{*}{ Person 2} & Moving in & 21 & 4 & 9 & 1 & $84 \%$ & $96 \%$ & $86 \%$ \\
\hline & Moving out & 20 & 5 & 7 & 3 & $80 \%$ & $87 \%$ & $77 \%$ \\
\hline \multirow[t]{2}{*}{ Person 3} & Moving in & 18 & 7 & 7 & 3 & $72 \%$ & $86 \%$ & $71 \%$ \\
\hline & Moving out & 19 & 6 & 8 & 2 & $76 \%$ & $91 \%$ & $77 \%$ \\
\hline \multirow[t]{2}{*}{ Person 4} & Moving in & 19 & 6 & 7 & 3 & $76 \%$ & $86 \%$ & $74 \%$ \\
\hline & Moving out & 20 & 5 & 8 & 2 & $80 \%$ & $91 \%$ & $80 \%$ \\
\hline \multirow[t]{2}{*}{ Person 5} & Moving in & 20 & 5 & 8 & 2 & $80 \%$ & $91 \%$ & $80 \%$ \\
\hline & Moving out & 22 & 3 & 8 & 2 & $88 \%$ & $92 \%$ & $86 \%$ \\
\hline \multirow[t]{2}{*}{ Person 6} & Moving in & 21 & 4 & 8 & 2 & $84 \%$ & $91 \%$ & $83 \%$ \\
\hline & Moving out & 20 & 5 & 8 & 2 & $80 \%$ & $91 \%$ & $80 \%$ \\
\hline \multirow[t]{2}{*}{ Person 7} & Moving in & 18 & 7 & 8 & 2 & $72 \%$ & $90 \%$ & $74 \%$ \\
\hline & Moving out & 18 & 7 & 9 & 1 & $72 \%$ & $95 \%$ & $77 \%$ \\
\hline \multirow[t]{2}{*}{ Person 8} & Moving in & 21 & 4 & 9 & 1 & $84 \%$ & $96 \%$ & $86 \%$ \\
\hline & Moving out & 21 & 4 & 8 & 2 & $84 \%$ & $91 \%$ & $83 \%$ \\
\hline \multirow[t]{2}{*}{ Person 9} & Moving in & 22 & 3 & 8 & 2 & $88 \%$ & $92 \%$ & $86 \%$ \\
\hline & Moving out & 18 & 7 & 8 & 2 & $72 \%$ & $90 \%$ & $74 \%$ \\
\hline \multirow[t]{2}{*}{ Person 10} & Moving in & 20 & 5 & 8 & 2 & $80 \%$ & $91 \%$ & $80 \%$ \\
\hline & Moving out & 19 & 6 & 9 & 1 & $76 \%$ & $95 \%$ & $80 \%$ \\
\hline Overall (TPD) & Moving in & & & & & $80 \pm 5 \%$ & $91 \pm 3 \%$ & $80 \pm 5 \%$ \\
\hline Using PF & Moving out & & & & & $79 \pm 5 \%$ & $91 \pm 2 \%$ & $80 \pm 3 \%$ \\
\hline Overall (TPD) & Moving in & & & & & $2 \pm 3 \%$ & $5 \pm 6 \%$ & $2 \pm 2 \%$ \\
\hline Using Scene Analysis & Moving out & & & & & $5 \pm 4 \%$ & $11 \pm 8 \%$ & $5 \pm 4 \%$ \\
\hline Overall (TD) & Moving in & & & & & $100 \%$ & $100 \%$ & $100 \%$ \\
\hline Using PF & Moving out & & & & & $100 \%$ & $100 \%$ & $100 \%$ \\
\hline Overall (TD) & Moving in & & & & & $100 \%$ & $100 \%$ & $100 \%$ \\
\hline Using Scene Analysis & Moving out & & & & & $100 \%$ & $100 \%$ & $100 \%$ \\
\hline
\end{tabular}

row and flag that partition as shown in Fig. 6(b)(ii). Finally, we compare this result with the ground truth to evaluate the accuracy of our TPD algorithm. In this example the the ground truth for Straight-in to Straight-out is given in Fig. 6(b)(iii).

\subsection{Statistical Analysis}

In this study, we evaluated the performance of both TPD and TD algorithms by determining: i) Recall = True Positives / (True Positives + False Negatives) and ii) Precision $=$ True Positives / (True Positives + False Positives); and iii) Accuracy $=$ True Positives + True Negatives / (True Negatives + True Positives + False Positives + False Negatives).

Since we are interested in determining if the supervised environment is similar to the unsupervised environment, we evaluated the if the results in supervised environment is statistically significantly different from the unsupervised environment. We used a two-tailed t-test where statistical significance was at $\mathrm{p}$-values $<0.05$.

\subsubsection{Traversing Path Detection Algorithm}

When evaluating the TPD algorithm: True positives (TP) were the paths that were correctly identified (e.g. Right-in to Left-out); True negatives (TN) were paths of no interest that were correctly identified (e.g. No-traversal in); False negatives (FN) (i.e. missed reads) were paths that were not identified due to lack of readings reported from the reader antennas (e.g. Left-in to Left-out is being reported as Notraversal out); and False positives (FPs) are other movements that were identified as a moving direction of interest.

\subsubsection{Traversing Direction Algorithm}

Here, we define the terms used in TD algorithm. TPs were movements that were correctly identified (e.g. moving out). TNs were movements of no interest that were correctly identified (e.g. No-traversal in). FNs were movements that were
Table 2: Performance of our PF based algorithms in an unsupervised environment and comparison with the scene analysis technique

\begin{tabular}{|c|c|c|c|c|c|c|c|c|}
\hline Person & Path & TP & FN & TN & FP & Recall & Precision & Accuracy \\
\hline \multirow[t]{2}{*}{ Person 1} & Moving in & 18 & 7 & 7 & 3 & $72 \%$ & $86 \%$ & $71 \%$ \\
\hline & Moving out & 17 & 8 & 7 & 3 & $68 \%$ & $85 \%$ & $69 \%$ \\
\hline \multirow{2}{*}{ Person 2} & Moving in & 18 & 7 & 8 & 2 & $72 \%$ & $90 \%$ & $74 \%$ \\
\hline & Moving out & 17 & 8 & 6 & 4 & $68 \%$ & $81 \%$ & $66 \%$ \\
\hline \multirow[t]{2}{*}{ Person 3} & Moving in & 17 & 8 & 7 & 3 & $68 \%$ & $85 \%$ & $69 \%$ \\
\hline & Moving out & 18 & 7 & 8 & 2 & $72 \%$ & $90 \%$ & $74 \%$ \\
\hline \multirow{2}{*}{ Person 4} & Moving in & 18 & 7 & 7 & 3 & $72 \%$ & $86 \%$ & $71 \%$ \\
\hline & Moving out & 16 & 9 & 8 & 2 & $64 \%$ & $89 \%$ & $69 \%$ \\
\hline \multirow[t]{2}{*}{ Person 5} & Moving in & 18 & 7 & 7 & 3 & $72 \%$ & $86 \%$ & $71 \%$ \\
\hline & Moving out & 16 & 9 & 8 & 2 & $64 \%$ & $89 \%$ & $69 \%$ \\
\hline \multirow[t]{2}{*}{ Person 6} & Moving in & 18 & 7 & 7 & 3 & $72 \%$ & $86 \%$ & $71 \%$ \\
\hline & Moving out & 19 & 6 & 7 & 3 & $76 \%$ & $86 \%$ & $74 \%$ \\
\hline \multirow[t]{2}{*}{ Person 7} & Moving in & 18 & 7 & 7 & 3 & $72 \%$ & $86 \%$ & $71 \%$ \\
\hline & Moving out & 17 & 8 & 7 & 3 & $68 \%$ & $85 \%$ & $69 \%$ \\
\hline \multirow[t]{2}{*}{ Person 8} & Moving in & 16 & 9 & 8 & 2 & $64 \%$ & $89 \%$ & $69 \%$ \\
\hline & Moving out & 17 & 8 & 6 & 4 & $68 \%$ & $81 \%$ & $66 \%$ \\
\hline \multirow[t]{2}{*}{ Person 9} & Moving in & 15 & 10 & 7 & 3 & $60 \%$ & $83 \%$ & $63 \%$ \\
\hline & Moving out & 18 & 7 & 8 & 2 & $72 \%$ & $90 \%$ & $74 \%$ \\
\hline \multirow[t]{2}{*}{ Person 10} & Moving in & 16 & 9 & 6 & 4 & $64 \%$ & $80 \%$ & $63 \%$ \\
\hline & Moving out & 16 & 9 & 7 & 3 & $64 \%$ & $84 \%$ & $66 \%$ \\
\hline $\begin{array}{l}\text { Overall (TPD) } \\
\text { Using PF }\end{array}$ & $\begin{array}{l}\text { Moving in } \\
\text { Moving out }\end{array}$ & & & & & $\begin{array}{l}69 \pm 4 \% \\
68 \pm 4 \%\end{array}$ & $\begin{array}{l}86 \pm 3 \% \\
86 \pm 3 \%\end{array}$ & $\begin{array}{l}69 \pm 4 \% \\
69 \pm 3 \%\end{array}$ \\
\hline $\begin{array}{c}\text { Overall (TPD) } \\
\text { Using Scene Analysis }\end{array}$ & Moving in & & & & & $4 \pm 3 \%$ & $8 \pm 6 \%$ & $4 \pm 3 \%$ \\
\hline$\frac{\text { Using Scene Analysis }}{\text { Oyerall (TD) }}$ & Moving out & & & & & $3 \pm 2 \%$ & $6 \pm 5 \%$ & $2 \pm 2 \%$ \\
\hline $\begin{array}{c}\text { Overall (TD) } \\
\text { Using PF }\end{array}$ & $\begin{array}{l}\text { Moving in } \\
\text { Moving out }\end{array}$ & & & & & $\begin{array}{l}100 \% \\
100 \%\end{array}$ & $\begin{array}{l}100 \% \\
100 \%\end{array}$ & $\begin{array}{l}100 \% \\
100 \%\end{array}$ \\
\hline Overall (TD) & Moving in & & & & & $100 \%$ & $100 \%$ & $100 \%$ \\
\hline Using Scene Analysis & Moving out & & & & & $87 \%$ & $100 \%$ & $91 \%$ \\
\hline
\end{tabular}

not identified (i.e. moving out is not being reported). FPs are other movements that were identified as a moving direction of interest (e.g. No-traversal in is being identified as Moving out).

\subsection{Results}

The results from Table 1 and Table 2 show that our TD algorithm was able to identify the tag traversal direction with $100 \%$ recall, precision and accuracy in both, supervised and unsupervised environments.

Although the mean performance values for TPD were higher in the supervised environment compared to the unsupervised environment, the difference is performance is not significantly different $(\mathrm{p}<0.01)$. Evaluating our TPD algorithms in both environments show that precision is $\geq 86 \%$ and is noticeably higher than recall and accuracy which is $\geq 68 \%$ and $\geq 69 \%$, respectively, for both moving in and moving out paths. This is because the number of FPs are comparatively lower than the number of FNs and hence the algorithms miss identifying complete paths. This is mostly due to missing readings in as a result of the tag over the shoulder being shadowed by a persons head.

Detecting the path used while eloping had $\leq 9 \%$ false alarm rate (chance of having incorrect path estimation such as Right-in to Left-out as Left-in to Right-out) in the supervised environment and $\leq 14 \%$ false alarm rate in the unsupervised environment. Consequently, as expected, there are less false alarms in the supervised environment compared to the unsupervised environment. Also, from Fig. 6(d) it is clear that the overall performance (mean recall, precision and accuracy) of the unsupervised environment is lower than the results of the supervised environment, because we used the same rssi_map for both the environments. The actual rssi_map was generated from the supervised environment and therefore the map was able to model measurement noise more accurately for the supervised environment than for the unsupervised environment. 
Next, we compare the results of scene analysis from Table 1 and Table 2. As expected, our TPD algorithm outperformed the baseline approach formulated using scene analysis to identify the traversal path used by a tag bearer. This is because, our PF based TPD algorithm is capable of continuously estimating the location of the tag bearer in a nonlinear and dynamic system using noisy RSSI measurements, in contrast, scene analysis technique simply compares the real-time rssi value with the collected rssi_map to infer the person's current location.

Comparing results for TD using scene analysis in the supervised environment with that of PF based TD shows that both approaches perform equally well. However, comparing the algorithms in the unsupervised environment show that the recall (87\%) and accuracy (91\%) results for scene analysis technique are lower in the moving out path. This is due to the missed read occurrences in the Right-in to Rightout path caused by the head obstructing the tag which is attached to the right shoulder from being interrogated by any of the deployed four antennas that are to the left of the person. The supervised environment has a wall located approximately $1.5 \mathrm{~m}$ from the setup and reflections from the wall contributed to illuminating the tag on the right shoulder while participants were in zone_C. Hence the scene analysis technique was able to predict the direction of the tag bearer in the supervised environment. However, in the unsupervised environment the adjacent wall was located $6 \mathrm{~m}$ from the right side of the setup and therefore, due to the obstruction caused by the head, there were only a few readings beyond zone_B in the Right-in to Right-out path. These missing readings resulted in $\mathrm{FN}$ in the evaluation of TD. In contrast, our TD algorithm is capable of predicting a persons future location in the presence of missing reads based on the motion model that describe how the state evolves over time and thus able to achieve better performance.

In particular, comparing the data presented in Table 1 and Table 2 we can also observe that the results for Person 4, Person 5, Person 8, Person 9 and Person 10 in the Table 2 have the lowest TPs compared to other participants. A possible reasons for the increased occurrences of FNs is the tag shifting position over the shoulder as a consequence of loosely worn clothing during the trial resulting in RSSI measurements that cannot be correctly filtered using the sensor model based on our measured rssi_map. On further investigation of the cause for the lowest number of TPs recorded for Person 9, we observed that measured RSSI values for Person 9 was lower compared to other participants. Person 9 was in fact the shortest participant (height: $153 \mathrm{~cm}$ ) among the 14 persons that participated in the trial. A possible reason for the increased number of FNs is the limitation of the measured sensor model (rssi_map) to adequately relate the location of the person to the measure RSSI values since the rssi_map was generated using a much more taller person with height of $170 \mathrm{~cm}$.

We also evaluated the effect of a tag bearer's traversing speed on the accuracy of our algorithms. We varied the walking speed from $0.18 \mathrm{~km} / \mathrm{h}(0.05 \mathrm{~m} / \mathrm{s})$ to $9 \mathrm{~km} / \mathrm{h}(2.5$ $\mathrm{m} / \mathrm{s}$ ). As shown in Fig. 6(c) walking speed had some impact on the accuracy. When the speed was too slow such as from $0.18 \mathrm{~km} / \mathrm{h}$ to $0.9 \mathrm{~km} / \mathrm{h}$ there is a small reduction (down to $78 \%$ ), in the accuracy of the TPD algorithm. This is because our motion model is initialised with a constant speed with additive increases or multiplicative decreases to adapt to the walking speed of the tag bearer over several iterations. Therefore, the first few iterations may not accurately model the speed of the tag bearer and consequently resulted in poor location estimates. However, our TD algorithm was able to, with $100 \%$ accuracy, determine the tag direction with walking speeds less than $7.2 \mathrm{~km} / \mathrm{h}$, beyond which the accuracy fell slightly lower to $99 \%$. Although, walking speed had some impact on the accuracy of our algorithms, our results were consistent in the normal walking speed (approx. $4.5 \mathrm{~km} / \mathrm{h}$ to $5.25 \mathrm{~km} / \mathrm{h}$ ) according to mean gait speed reported in [7] for people aged 40 and above.

\section{CONCLUSIONS}

We developed a novel, robust, real-time system that can accurately detected the traversal path used by the tag bearer and the tag bearer's moving direction such as moving out and moving in. In our study we used minimum number of instruments i.e a single, low cost passive RFID tag and four RFID antennas to achieve a $100 \%$ accuracy while detecting tag traversal direction, $\leq 9 \%$ false alarm on traversal path detection and $\leq 20 \%$ misses.

Our approach is a significant enhancement when compared to the existing approaches and the elimination of false alarms and misses (i.e. for TD) and the relatively accurate estimations of paths travelled is likely to results in higher levels of acceptance among caregivers since we are able to comprehensively address frustrations from false alarms while reliably identifying the eloping direction. Our algorithm can also be generalised to solve other problems such as detect goods that are travelling in and out of a warehouse however, there may be limitations in the performance depending on the speed at which goods are in transit.

Even though, our algorithm performed well throughout the study, certain paths results such as Right-in to Right-out frequently performed poorly due to the higher occurrences of missed reads. TD performance in the unsupervised environment suggests that it is possible to use apply a sensor model developed in a similar context in a difference environment. However, the performance of the algorithm with respect of heights of people still needs to be investigated.Also, we did not investigate the accuracy of our algorithms with multiple participants and we have left this as future work. We are currently looking at developing a generic sensor model instead of scene analysis, so that it can be directly implemented in new environments to achieve similar performance to that possible in a supervised environment.

\section{ACKNOWLEDGMENTS}

This research was supported by a grant from the Hospital Research Foundation (THRF) in South Australia and the Australian Research Council (DP130104614).

\section{REFERENCES}

[1] Alzheimer's facts and figures. www.alz.org, 10/06/2014.

[2] The department of health: Dementia. www.health.gov.au/dementia, 10/06/2014. 
[3] Fujitsu laundry tags. www.atlasrfidstore.com/rfid_laundry_tags_s, $17 / 10 / 2014$

[4] Azheimer's association and others. 2013 alzheimer's disease facts and figures. Alzheimer's 8 Dementia: the Journal of the Alzheimer's Association, 9(2):208, 2013.

[5] M. S. Arulampalam, S. Maskell, N. Gordon, and T. Clapp. A tutorial on particle filters for online nonlinear/non-gaussian bayesian tracking. IEEE Tran. on Signal Processing,, 50(2):174-188, 2002.

[6] M. A. Aud. Dangerous wandering: elopements of older adults with dementia from long-term care facilities. American Journal of Alzheimer's Disease and Other Dementias, 19(6):361-368, 2004.

[7] R. W. Bohannon. Comfortable and maximum walking speed of adults aged 20-79 years: reference values and determinants. Age and Ageing, 26(1):15-19, 1997.

[8] M. Bouet and A. L. Dos Santos. RFID tags: Positioning principles and localization techniques. In Wireless Days, 2008. 1st IFIP, pages 1-5, 2008.

[9] P. H. Cole and D. C. Ranasinghe. Networked RFID systems and lightweight cryptography. London, UK: Springer. doi, 10:978-3, 2008.

[10] M. Goller, C. Feichtenhofer, and A. Pinz. Fusing RFID and computer vision for probabilistic tag localization. In IEEE Int. Conf. on RFID, Orlando, USA, 2014.

[11] D. Hahnel, W. Burgard, D. Fox, K. Fishkin, and M. Philipose. Mapping and localization with RFID technology. In Proc. of IEEE Int. Conf. on Robotics and Automation, 2004. ICRA'04., volume 1, pages 1015-1020. IEEE, 2004.

[12] R. Hope and C. G. Fairburn. The nature of wandering in dementia: A community-based study. Int. Journal of Geriatric Psychiatry, 5(4):239-245, 1990.

[13] Y. Jian, Y. Wei, and Y. Zhang. Estimating the direction of motion based on active RFID. In 5th Int. Conf. on New Trends in Information Science and Service Science (NISS), volume 2, pages 286-290. IEEE, 2011.

[14] D. Joho, C. Plagemann, and W. Burgard. Modeling RFID signal strength and tag detection for localization and mapping. In IEEE Int. Conf. on Robotics and Automation, 2009. ICRA'09., pages 3160-3165. IEEE, 2009.

[15] T. Keller, F. Thiesse, A. Ilic, and E. Fleisch. Decreasing false-positive rfid tag reads by improved portal antenna setups. In 3rd Int. Conf. on the Internet of Things (IOT), pages 99-106. IEEE, 2012.

[16] S. C. Kim, Y. S. Jeong, and S.-O. Park. RFID-based indoor location tracking to ensure the safety of the elderly in smart home environments. Personal and ubiquitous computing, 17(8):1699-1707, 2013.

[17] M. Lehtonen, D. Ostojic, A. Ilic, and F. Michahelles. Securing rfid systems by detecting tag cloning. In Pervasive Computing, pages 291-308. Springer, 2009.

[18] F. Miskelly. A novel system of electronic tagging in patients with dementia and wandering. Age and Ageing, 33(3):304-306, 2004.

[19] L. M. Ni, Y. Liu, Y. C. Lau, and A. P. Patil. Landmarc: indoor location sensing using active RFID. Wireless Networks, 10(6):701-710, 2004.
[20] Y. Oikawa. Evaluation of tag moving direction detection in a UHF RFID gate system. Journal of Circuits, Systems, and Computers, 21(06), 2012.

[21] D. C. Ranasinghe, R. L. Shinmoto Torres, A. P. Sample, J. R. Smith, K. Hill, and R. Visvanathan. Towards falls prevention: A wearable wireless and battery-less sensing and automatic identification tag for real time monitoring of human movements. In 34th Int. IEEE EMBS Conference, San Diego, USA., 2012.

[22] S. S. Saad and Z. S. Nakad. A standalone RFID indoor positioning system using passive tags. IEEE Trans. on Industrial Electronics, 58(5):1961-1970, 2011.

[23] R. Sankarkumar, D. C. Ranasinghe, and T. Sathyan. A highly accurate method for managing missing reads in RFID enabled asset tracking. In 10th Int. Conf. on Mobile and Ubiquitous Systems, Tokyo, Japan, 12/2013 2013.

[24] R. Sankarkumar, D. C. Ranasinghe, and T. Sathyan. A highly accurate and scalable approach for addressing location uncertainty in asset tracking applications. In IEEE Int. Conf. on RFID, Orlando, USA, 2014.

[25] F. Sposaro. A geriatric suite of medical applications for android powered devices. Electronic Theses, Treatises and Dissertations. Paper 5199, 2012.

[26] W. Thiesand and L. Bleiler. Alzheimer's disease facts and figures. Alzheimer's 85 Dementia: the Journal of the Alzheimer's Association, 7(2):208-244, 2011.

[27] J. Wan, M. J. O'Grady, and G. M. O'Hare. Towards holistic activity modeling and behavioral analyses. 6th Int. workshop on Ubiquitous health and wellness, Newcastle, UK, 2012.

[28] J. Wang and D. Katabi. Dude, where's my card?: RFID positioning that works with multipath and non-line of sight. In Proc. of the Conf. on SIGCOMM 2013, pages 51-62. ACM, 2013.

[29] J. Yu, W.-S. Ku, M.-T. Sun, and H. Lu. An RFID and particle filter-based indoor spatial query evaluation system. In Proc. of the 16th Int.Conf. on Extending Database Technology, pages 263-274. ACM, 2013.

[30] Y. Zhang, M. G. Amin, and S. Kaushik. Localization and tracking of passive RFID tags based on direction estimation. Int. Journal of Antennas and Propagation, 2007.

[31] Y. Zhao, Y. Liu, and L. M. Ni. Vire: Active RFID-based localization using virtual reference elimination. In Int. Conf. on Parallel Processing, ICPP 200\%., pages 56-56. IEEE, 2007.

[32] M. Zhou and D. C. Ranasinghe. A novel approach for addressing wandering off elderly using low cost passive RFID tags. In Int. Conf. on Mobile and Ubiquitous Systems, Tokyo, Japan, 2013. 